PAEDIATRIC LUNG DISEASE

\title{
Safety of endobronchial biopsy in 170 children with chronic respiratory symptoms
}

\author{
P S Salva, C Theroux, D Schwartz
}

Thorax 2003;58:1058-1060

See end of article for authors' affiliations .....................

Correspondence to: Dr Paul S Salva, 780 Chestnut St Suite \#11, Springfield, MA 01107 , USA; pedipulm@cox.net

Received 6 January 2003 Accepted for publication 27 July 2003
Background: There is a paucity of bronchial biopsy data in children. A major limitation is concern over the safety of the procedure. This paper reports the results of efforts to develop a method that is safe and provides adequate specimen for evaluation.

Methods: 170 children aged 2.5 to 16 years with chronic respiratory symptoms were studied under general anaesthesia in an outpatient surgery setting. Bronchoalveolar lavage and biopsies were obtained using a $4.9 \mathrm{~mm}$ flexible bronchoscope through a laryngeal mask airway. At least three bioipsies were taken.

Results: No patient required topical adrenaline to control bleeding, nor was there a change in the state of any of the patients. There were no episodes of pneumothorax, haemoptysis, pneumonia, or significant fever. All children less than four years old received a single dose of antibiotic intravenously after the procedure. The average length of time for the procedure was 12 minutes (range 6 to 27). Recovery time averaged 90 minutes. The limiting factor was the ability of the child's airway to accomodate the bronchoscope.

Conclusions: This report should encourage clinicians to incorporate endobronchial biopsy into the evaluation of children with difficult respiratory problems.
$\mathrm{E}$ valuating young children with chronic respiratory problems can be challenging. In young children, the history is obtained secondhand, from the parents, while older children may not be able to describe their symptoms in ways understood by adults. Obtaining spirometric data is limited by age and is dependent on technique and cooperation. Other studies-such as skin or RAST testing, immune and gastrointestinal studies, and radiography-provide valuable information but may not allow a definitive diagnosis to be made. There has been increasing discussion about the need for bronchial biopsy in children for diagnostic purposes and to learn about the earliest phases of respiratory illnesses. ${ }^{1-3}$

Until recently, ${ }^{4-7}$ there were very few endobronchial biopsy data in children. The major limitations of the procedure in children are a fear of complications such as haemorrhage or pneumothorax, a hesitancy to undertake an invasive procedure, and the uncertainty that it would provide meaningful data in the absence of published data for comparison. The first priority, however, is to develop techniques that are safe.

Two groups $s^{4}$ have recently reported endobronchial biopsy data in children. One study was limited to 10 children, all studied by rigid bronchoscopy, ${ }^{4}$ and reported findings similar to those in adults. The second study ${ }^{5}$ used both rigid and flexible bronchoscopy, addressed the safety and ethics of obtaining biopsies, but did not discuss the clinical findings. In this report we describe our experience in obtaining endobronchial biopsies employing a flexible fibreoptic bronchoscopy/laryngeal mask airway technique in an outpatient setting in 170 children. We document the safety of the procedure in children as young as 2.5 years of age. Clinical findings will be reported separately.

\section{METHODS}

Institutional review board approval was obtained to collect data prospectively on children presenting for bronchoscopy. Children were referred for bronchoscopy after a thorough evaluation did not yield a diagnosis, or after medical management did not produce an adequate improvement in their clinical state. ${ }^{8}$ The asthmatic children were taking high doses of inhaled corticosteroid (>800 $\mu \mathrm{g}$ /day), long acting bronchodilators, and a leukotriene antagonist, yet continued to require daily short acting bronchodilator treatment and frequent courses of systemic steroids (more than four a year). The procedure was explained to the patient and family verbally, and a written description was provided prospectively by the paediatric pulmonologist. On admission to the outpatient procedure suite, the child was evaluated by a paediatric anaesthesiologist. Written informed consent was obtained.

All patients had been barred from taking solids or liquids for at least eight hours. Most children underwent induction of general anaesthesia with sevoflurane in oxygen and nitrous oxide using a mask technique, with a parent present. After controlled loss of consciousness, routine monitoring of ECG, blood pressure, end tidal carbon dioxide, and pulse oximetry (Novametrix Medical Systems, Wallingford, Connecticut, USA) was undertaken. A peripheral intravenous catheter was then inserted. Older children had the option of having an intravenous line inserted while they were awake and then, after placement of monitors, undergoing intravenous induction with propofol, $2-3 \mathrm{mg} / \mathrm{kg}$. During bronchoscopy, ventilation was maintained by the anaesthesiologist giving positive pressure through the anaesthesia circuit, with either controlled or assisted breaths. The adequacy of ventilation was monitored by inspecting the end tidal $\mathrm{CO}_{2}$ monitor as well as by visual inspection of chest excursion. Sevoflurane in $100 \%$ oxygen with or without intermittent intravenous propofol $(0.5$ to $1.0 \mathrm{mg} / \mathrm{kg})$ was used for maintenance of anaesthesia.

A laryngeal mask airway (LMA North America Inc, Los Angeles, California, USA) was used in all instances. The bronchoscopies were done by one investigator (PSS). A flexible fibreoptic bronchoscope (Olympus BF type P20D or type P40, $4.9 \mathrm{~mm}$ outside diameter) was introduced through the mask airway. One to three millilitres of $1 \%$ aqueous 
lignocaine (lidocaine) were applied to the vocal cords. The bronchoscope was advanced to the level of the carina and both sides of the bronchial tree were inspected. The right middle lobe was lavaged with normal saline $(20-50 \mathrm{ml})$ unless the visual examination showed a macroscopic abnormality elsewhere. The lavage aspirate was divided into three aliquots: culture for aerobic bacteria, cell count and differential, and staining for lipid laden macrophages.

Endobronchial biopsies were taken from various sites of the right bronchial tree at the level of the third branch in children less than 10 years old and at the third or fourth branch in the older children, using Bard Precisor BRONCHO coated disposable biopsy forceps (Bard Endoscopic Technologies, Billerica, Massachusetts, USA). A minimum of three biopsies was taken. The specimens were placed in formalin and processed for staining with haematoxylin and eosin. Biopsies from the left side of the bronchial tree were taken only if the visual examination showed a macroscopic abnormality.

Once the procedure was completed, patients were taken to a recovery area with face mask oxygen and their ECG, blood pressure, and pulse were monitored until they were sitting up and tolerating fluids, typically about 90 minutes. They were then discharged from the endoscopy suite. Patients of less than four years of age were given either ampicillin $(50 \mathrm{mg} / \mathrm{kg})$ or cefazolin $(25 \mathrm{mg} / \mathrm{kg})$ as a single intravenous dose after the procedure.

\section{RESULTS}

The mean age of the patients was 9.3 years (range 2.5 to 16). The most common indication for the procedure was chronic cough. Other conditions were asthma, recurrent pneumonia, focally abnormal breath sounds, bronchopulmonary dysplasia, haemoptysis, interstitial lung disease, and dyspnoea (table 1). Twenty nine patients (six girls, 23 boys) were less than five years old, 66 (32 girls, 34 boys) were between six and 10 years old, and 75 (39 girls, 36 boys) were 11 to 16 years old. Regarding ethnicity, 121 were white (57 girls, 64 boys), 22 were black (six girls, 16 boys), 25 were hispanic ( 13 girls, 12 boys), and two were Asian (one girl, one boy). The procedure took an average of 12 minutes (range 6 to 27). No patient required intubation, admission to the hospital, or change in status if they were already in the hospital. Length of hospital stay was not affected. Fluctuations in oxygen saturation and end tidal $\mathrm{CO}_{2}$ occurred in all patients. Only one patient experienced a prolonged oxygen desaturation $(<85 \%)$. He was found to have a right bronchial stenosis. The desaturation resolved with a brief interruption of the procedure to allow for assisted positive pressure breathing.

There was local bleeding at the biopsy sites; this was minimal and never required intervention with topical adrenaline. There were no episodes of haemoptysis, pneumothorax, pneumonia, or clinically significant fever. No patient experienced laryngospasm or bronchospasm

\begin{tabular}{lll}
$\begin{array}{l}\text { Table } \mathbf{1} \\
\text { patients }\end{array}$ & Indication for bronchoscopy and the sex of the \\
\hline Indication & $\mathbf{n}$ & $\mathrm{F} / \mathrm{M}$ \\
\hline Cough & 98 & $46 / 52$ \\
Asthma & 39 & $16 / 23$ \\
Recurrent pneumonia & 15 & $7 / 8$ \\
Focal breath sounds & 6 & $3 / 3$ \\
BPD & 3 & $1 / 2$ \\
Haemoptysis & 3 & $2 / 1$ \\
ILD & 2 & $0 / 2$ \\
Dyspnoea & 4 & $2 / 2$ \\
\hline
\end{tabular}

BPD, bronchopulmonary dysplasia; F, female; ILD, interstitial lung disease; $M$, male. requiring bronchodilator treatment, or stridor requiring nebulised racemic adrenaline.

The size of the biopsy sample was adequate for microscopic evaluation. The families were instructed to call the pulmonologist (PSS) with concerns, and a phone follow up took place the next morning. Office follow up occurred seven to 10 days later, with the families consistently reporting an uneventful postprocedural course.

The bronchoscopic findings led to a specific diagnosis or change in management in $82 \%$ of the children studied. Of the 39 asthmatic children studied, secondary morbidities were found in 20. Structural anomalies were found in five, airway malacia in three, aspiration in seven, and bacterial bronchitis in five.

\section{DISCUSSION}

The decision to attempt mucosal biopsies arose from our experience in removing pulmonary foreign bodies and our frustration in not being able at times to make a definitive diagnosis by other means. During foreign body removal, bleeding occurred on many occasions, with visible disruption of the mucosa because the object was impacted or had a jagged surface. The mucosa was oedematous, hyperaemic, often macerated, and bled easily. However, after removal, the children uniformly did well, without haemoptysis, desaturation, or pneumothorax. Following to this experience, and starting in 1997, we felt that endobronchial biopsy could be incorporated into our evaluation of children with persistent respiratory symptoms with reasonable safety. Endobronchial biopsy was undertaken after a thorough evaluation-which included radiographic, immune, allergic, and spirometric studies, pH probe, and upper endoscopy-did not yield a diagnosis, and after aggressive medical management did not provide adequate improvement in the child's clinical state.

We feel that general anaesthesia is the method of choice for children undergoing this procedure, as opposed to sedation with local anaesthesia applied to the respiratory mucosa. Unlike the latter, general anaesthesia provides a near-motionless patient, which allows the procedure to proceed efficiently and safely. As the patient is still breathing spontaneously, an assessment of the dynamic properties of the respiration can be made. In addition, the child experiences little anxiety and has no recall. The laryngeal mask airway brings the bronchoscope directly to the laryngeal inlet, allowing inspection of the vocal cords and an unobstructed view of the trachea. This cannot be achieved with an endotracheal tube or a rigid bronchoscope.

The methods employed in our series vary considerably from other recent reports. In the series by Cokugras et al, ${ }^{4} 10$ children (aged five to 14 years) were examined by rigid bronchoscopy, with all drug treatments stopped for four weeks. In the series by Payne et al, 83 children, asthmatic and non-asthmatic (aged four to 17 years), were studied. In the asthmatic group, some were studied with flexible and others with rigid bronchoscopes. Most importantly, all asthmatic children received oral prednisolone $(2 \mathrm{mg} / \mathrm{kg} /$ day, maximum dose $40 \mathrm{mg}$ /day) for two weeks and nebulised salbutamol $(5 \mathrm{mg})$ immediately before the procedure.

In our series, patients were studied exclusively for clinical reasons and continued their prescribed drug treatment. They were not pretreated with systemic steroids or bronchodilators. All were examined with a flexible fibreoptic bronchoscope through a laryngeal mask airway and had lavage aspirates as well as mucosal biopsies taken. Lastly, patients as young as 2.5 years of age were studied successfully. The limiting factor was the child being big enough to accommodate the $4.9 \mathrm{~mm}$ bronchoscope. Younger children (six to 30 months) were studied with a smaller $(3.5 \mathrm{~mm})$ bronchoscope and forceps without complications. However, the 
biopsy specimen was inadequate for evaluation owing to its small size or because of crush artefact. Further attempts were abandoned after studying three patients.

We did not experience any episodes of clinically significant fever, in contrast to other published reports. ${ }^{9}{ }^{10}$ However, we did treat all children less than four years of age with a single dose of antibiotic intravenously after the procedure. This was incorporated into the procedure following our earlier experience of bronchial lavage on young children, wherein line with published reports-fever occurred frequently. Fever did not occur in children over four years of age unless purulent secretions were recovered. It occurred often in children less than four years old regardless of the physical appearance of the airway. Review of bronchial lavage culture data from the younger children showed that $12 \%$ were positive for bacteria: $>100000 \mathrm{cfu} / \mathrm{ml}$ of $S$ pneumoniae, $M$ catarrhalis, or non-typable $H$ influenza. Gram stains and differential cell counts on the lavage fluid were negative for squamous epithelial cells, making oral contamination unlikely. Other investigators ${ }^{11}$ have recently reported a similar incidence $(14.8 \%)$ of bacterial bronchitis in asthmatic children.

A debate has emerged about bronchial biopsy in children. ${ }^{12}$ The issues raised concern the ethics of performing invasive prospective studies and withdrawing therapeutic drugs beforehand. ${ }^{4}$ We also have concerns over this. The first relates to the classification of patients with moderate asthma. It is doubtful whether patients who really have moderate asthma could tolerate removal of anti-asthma treatment for four weeks without deteriorating. The fact that these patients did not deteriorate would suggest that they had mild disease or that they did not have asthma at all. That might explain the fact that eosinophils were seen in only two of 10 patients' biopsies. As these patients tolerated withdrawal of treatment, there was not even a good clinical reason to study them. In the second report, ${ }^{5}$ all asthmatic children were treated with oral steroids for two weeks beforehand at a dose commonly used to treat asthma exacerbations. The data collected in this circumstance are of little value in either the general sense or for the individual patients, as they do not reflect the patients' baseline clinical status.

Our data were collected solely for clinical reasons without any change in drug treatment. While broad conclusions about the biopsy findings are not possible owing to the variability in the patients' symptoms and drug treatment regimens, that was not the purpose of this report. Our aim was to document the safety of the procedure. The data show four things. The first is that bronchial biopsy is indeed safe and technically feasible in children as young as 2.5 years. Second, it is safe over a wide range of clinical conditions. Third, rigid bronchoscopy is not necessary. This should give pulmonologists confidence to perform the procedure themselves. Lastly, the data show that patients do not need to be pretreated routinely with systemic steroids. This should allow a more accurate assessment of their clinical situation.

The decision to undertake bronchoscopy on a child is not an easy one. The potential benefit has to outweigh the risk. For the moment, bronchoscopy with endobronchial biopsy should be reserved for those patients who have failed medical management and remain burdened by their condition.

\section{Conclusions}

This series shows that endobronchial biopsy is quite safe when done under general anaesthesia using a flexible bronchoscope and a laryngeal mask airway. We hope this report will encourage other practitioners to consider endobronchial biopsy in evaluating children with respiratory conditions that are hard to diagnose.

\section{ACKNOWLEDGEMENTS}

The authors thank Dr S Sanjar and Dr S Shrewsbury for their words of encouragement and advice.

\section{Authors' affiliations}

P S Salva, Pediatric Pulmonology of Western New England, Springfield, Massachusetts, USA

C Theroux, D Schwartz, Baystate Medical Center, Department of Anaesthesia, Springfield, Massachusetts, USA

\section{REFERENCES}

1 Bush A, Pohunek P. Brush biopsy and mucosal biopsy. Am J Resp Crit Care Med 2000;162:s18-22.

2 Ranganathan SC, Payne DNR, Jaffe A, et al. Difficult asthma: defining the problems. Pediatr Pulmonol 2001;31:114-20.

3 Jeffrey $\mathbf{P}$. Inflammation and remodeling in the adult and child with asthma. Pediatr Pulmonol 2001 ; (suppl 21):3-16.

4 Cokugras H, Akcakaya N, Seckin I, et al. Ultrastructural examination of bronchial biopsy specimens from children with moderate asthma. Thorax 2001;56:25-9.

5 Payne D, McKenzie SA, Stacey S, et al. Safety and ethics of bronchoscopy and endobronchial biopsy in difficult asthma. Arch Dis Child 2001;84:423-6.

6 Payne DNR, Adcock IM, Wilson NM, et al. Relationship between exhaled nitric oxide and mucosal eosinophilic inflammation in children with difficult asthma, after treatment with oral prednisone. Am J Resp Crit Care Med $2001 ; 164: 1376-81$.

7 Payne DNR, Rogers AV, Adelroth E, et al. Early thickening of the reticular basement membrane in children with difficult asthma. Am J Resp Crit Care Med 2003; 167:78-82.

8 Wood RE. Diagnostic and therapeutic procedures in pediatric pulmonary patients. In: Taussig LM, Landau LI, eds. Pediatric respiratory medicine. St Louis: CV Mosby, 1999:244-62.

9 Schellhase DE, Tamez JR, Menendez AA, et al. High fever after flexible bronchoscopy and bronchoalveolar lavage in noncritically ill immunocompetent children. Pediatr Pulmonol 1999;28:139-44.

10 Picard E, Schwartz S, Goldberg S, et al. A prospective study of fever and bacteremia after flexible fiberoptic bronchoscopy in children. Chest 2000; 117:573-7.

11 Just J, Fournier L, Momas I, et al. Clinical significance of bronchoalveolar eosinophils in childhood asthma. J Allergy Clin Immunol 2002;110:42-4.

12 Bush A, de Jongste JC, Carlsen K-H. Letter to the editor. Thorax 2003;58:187-8. 